\title{
Dynamics Research of rigid-flexible model of crank-rocker mechanism with Multi Clearance
}

\author{
Shao Jun BO ${ }^{1, a}$, Tian $\mathrm{Ci} \mathrm{CHEN}^{1}$ and Qing Huai YE ${ }^{2}$ \\ ${ }^{1}$ Institute of Mechanical Engineering Inner Mongolia University of Science and Technology,Baotou, Inner Mongolia, China \\ ${ }^{2}$ Baotou Steel Group Design Institute,Baotou, Inner Mongolia, China \\ Aerding Street on the 7th, Kundulun District,Baotou City, Inner Mongolia Province
}

\begin{abstract}
This paper takes the crank-rocker mechanism as the research object, purposed for the simulation of the motion pair with clearance utilizing the contact force model and the coulomb friction model, we build the virtual prototype of four-bar mechanism with clearance joints in the ADAMS. The article mainly contrasts the influence dynamics characteristics towards the mechanism multi-joint clearance between the rigid rocker and the flexible rocker . Considering the jointed arm' s flexibility, the four,five and six order modal of the rocker, generated by ANSYS as a neutral document, were imported into ADAMS for simulation. The result shows that Mechanism with four joint clearance increased the impact on the speed and the acceleration appeared greater fluctuation,the flexible rod has a buffer effect to the contact-impact forces,and the dynamic characteristics of fluctuations are improved.
\end{abstract}

Key Words: crank-rocker multi-joint clearance flexible body ADAMS dynamics characteristics

\section{Introduction}

With the high speed, overloading, the rapid development of precision mechanical and aerospace engineering, accurate prediction of mechanism motion demand is higher and higher. In most of the mechanical system, the mechanism of artifacts usually by kinematic pair connection. Due to error in the process of manufacture, assembly and mechanism motion produced by friction, wear, and the influence of such factors as movement exists between vice clearance. And the existence of the gap destroyed an ideal organization model, cause the collision of kinematic pair, cause the violent vibration of institutions, led to the decrease of the mechanical system stability and precision, and thus the efficiency and service life. In recent years, for mechanism with clearance, and there were many research of flexible components [1], but for considering four kinematic pair clearance and flexible connecting rod four-bar linkage study also is less. In this paper, based on two state model using coulomb friction model and collision model [2-4], and consider the connecting rod for the flexible body, including four crank rocker mechanism with clearance joints and soft model for the study of dynamics.

\footnotetext{
${ }^{a}$ Corresponding author: 15540180997@163.com
} 


\section{BUILT OF MODEL WITH MULTI-JOINT CLEARANCE}

As the existence of the joint clearance, the center distance between the shaft pin and sleeve is no longer zero, and resulted collision and friction of shaft pin and sleeve. There are three kinds states of shaft pin: free state, collision, Continuous contact. Center distance is e, $\mathrm{r}=\mathrm{R}_{\mathrm{i}}-R_{j}$, as shown in Fig. 1 .

When $\mathrm{e}<\mathrm{r}$, shaft pin is in a free state.

When $e>r$, the shaft pin and sleeve generate collision.

When the shaft pin and the sleeve in collision, the contact force decompose into the normal collision force $F_{N}$ and the tangential friction $F_{T}$.

In order to describe the rule of the collision between two objects, ADAMS software use the IMPACT function to solve the force of two objects at the time of the collision, and the motion rule of the two objects in the before collision and after collision. The function of the collision between two objects is equivalent to a nonlinear spring-damper model. As shown in Fig.2.

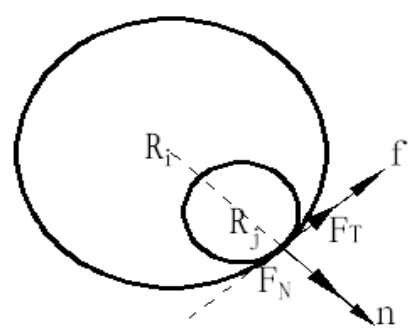

Fig 1. Normal and tangential contact force when the shaft pin and sleeve is in a collision.

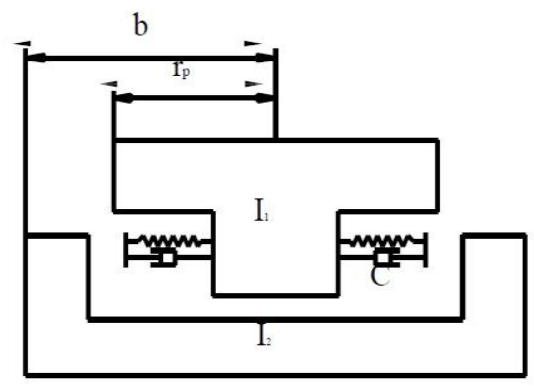

Fig 2. Figure4 Elastic damping model of impulse function.
The expression of the Impact function is as follows[5]:

$$
\begin{aligned}
& F_{\mathrm{N}}= \begin{cases}K \delta^{n}+\operatorname{Step}\left(\delta, 0,0, d_{\text {max }}, c_{\text {max }}\right) \frac{d \delta}{d t}, & \delta>0 \\
0, & \delta<0\end{cases} \\
& F_{T}=F_{N} \mu\left(v_{N}\right)
\end{aligned}
$$

$\mathrm{K}$ is stiffness coefficient, the fomula is as follows:

$$
\mathrm{K}=\frac{4}{3 \pi\left(\mathrm{h}_{i}+h_{j}\right)} R^{\frac{1}{2}}, R=\frac{R_{i} R_{j}}{R_{i}+R_{j}}, h_{k}=\frac{1-v_{k}^{2}}{\pi E_{k}}, k=i, j .
$$

$\mathrm{E}_{\mathrm{k},}, v_{k}, R_{k}$ represent Young's modulus, Poisson ratio and contact radius of the object. $\delta$ is the collision depth.Collision index $\mathrm{n}$ can reflect the level of material nonlinearity, normal metal materials takes the number of 1.5.In ADAMS, transient damping coefficient is the step function of collision depth, the value of $\mathrm{c}$ is determined by the Step function. Generally speaking, damping coefficient value take $0.1 \sim 1 \%$ of stiffness coefficient $\mathrm{K}[6]$, recommend $d_{\max }$ value is $0.01 \mathrm{~mm}$.Generally, friction coefficient data obtained from experiments, normal dynamic friction coefficient take 0.1 , static friction coefficient take 0.3 .

\section{DYNAMICS SIMULATION OF PLANAR FOUR-BAR MECHANISM WITH MULTI-JOINT CLEARANCE}

Fig.3 shows multi-joint clearance mechanism, put all parts material for steel.The four-bar mechanism parameters are as follows:

Crank $=105 \times 20 \times 5(\mathrm{~mm})$, Link $=272 \times 20 \times 5(\mathrm{~mm})$, 
Rocker $=175 \times 20 \times 5(\mathrm{~mm})$, Frame $=225 \times 20 \times 5(\mathrm{~mm}) ; \mathrm{k}$ $=2.53 \times 10^{5} \mathrm{~N} / \mathrm{mm}$, Maximum damping coefficient $c_{\text {max }}=253 \mathrm{~N} \times \mathrm{S} / \mathrm{m}$; Young's modulus $\mathrm{E}=207 \mathrm{Gpa}$,

Poisson's ratio $v_{i}=0.29$, the rotation of the crank at a constant speed $\omega=300 \mathrm{r} / \mathrm{min}$.In the crank and connecting rod, connecting rod and rocker, rocker arm and the frame, frame with motion pair with kinematic pair clearance between the crank, and then USES the ANSYS software component, the rocker to generate flexible body take flexible joystick four, five, six order modal system dynamics simulation, with a handle for the rigid rod when comparing the simulation results.

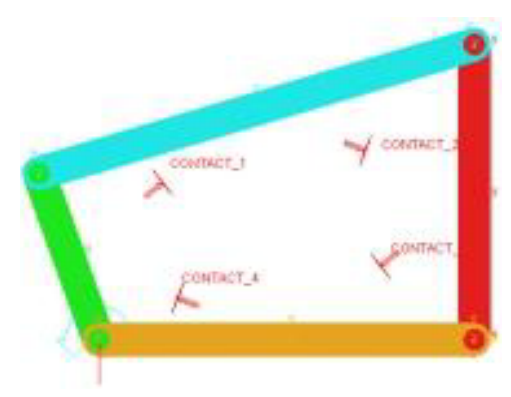

Figure3. Crank-rocker mechanism ADAMS.
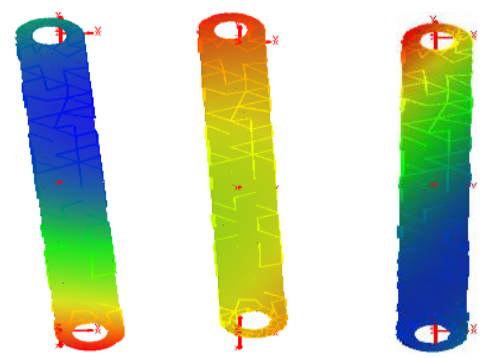

Figure4. Four,five and six modal of flexible rocker.

The simulation results and analysis.Figure 5 and figure 6 respectively a handle for the rigid and flexible connecting rod and rocker speed curve. Agencies began to work, two kinds of condition occurred on the connecting rod and a handle speed curve of a certain fluctuation, but the joystick is flexible, the speed is less volatile. At a later time, compared with the rigid rocker, flexible rocker rocker and connecting rod overall more smoothly, less fluctuations, shows that the flexible factors counter collision between shaft and shaft sleeve have buffer action.

Figure 7 and figure 8 respectively a handle for the rigid and flexible connecting rod and rocker speed curve. Rigid for analysis, rocker, connecting rod and rocker acceleration to the existence of the gap significantly in unstable state, shaft and shaft sleeve is in a state of collision and separation. When rocker for the flexible connecting rod and a handle fluctuates significantly decreases, and the acceleration amplitude greatly reduced, curve fluctuations more smoothly, also shows that the collision of flexible factor counter shaft and shaft sleeve have buffer action.

Figure 9 and 10 respectively a handle for the rigid and flexible component pin shaft and shaft sleeve contact between the collision force curve. Diagram curve clearly shows flexible joystick and shaft sleeve absorbs the shock, the collision has obvious cushioning, flexible factors to contain much clearance between the crank rocker mechanism motion state is very good, make body more close to ideal state, reduce the rigid adverse impacts on the state of motion artifacts. 

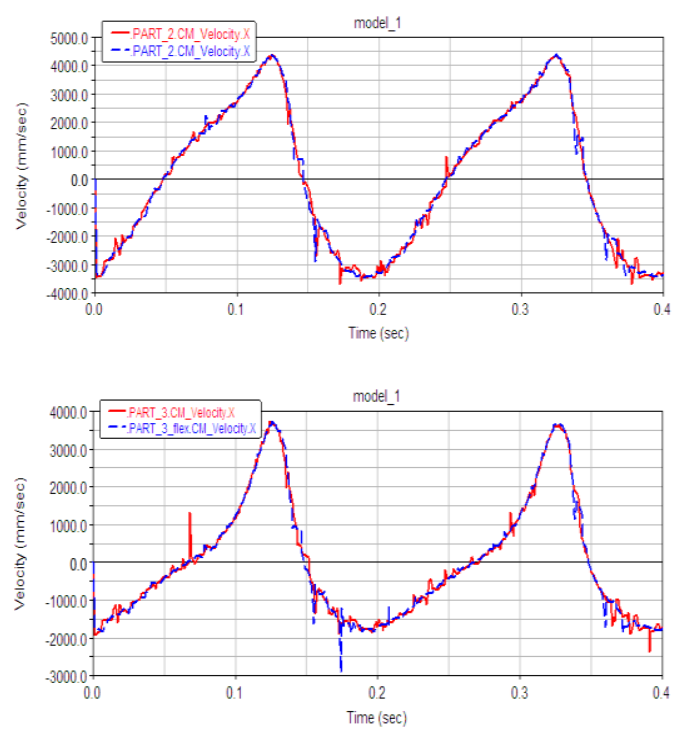

Figure5. Link X-axis speed in the case of the rigid and flexible rocker.

Figure6. Rocker X-axis speed in the case of the rigid and flexible rocker.
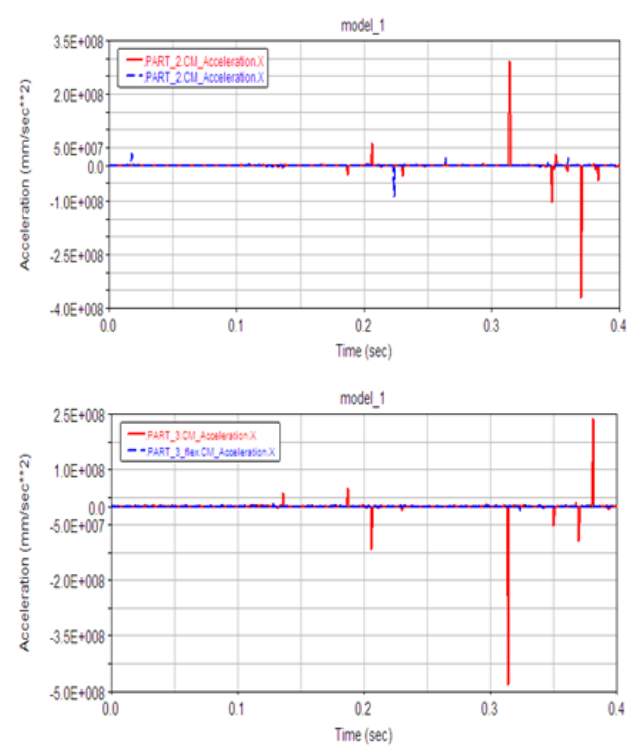

Figure7. Link X-axis acceleration in the case of the rigid and flexible rocker.

Figure8. Rocker X-axis acceleration in the case of the rigid and flexible rocker.
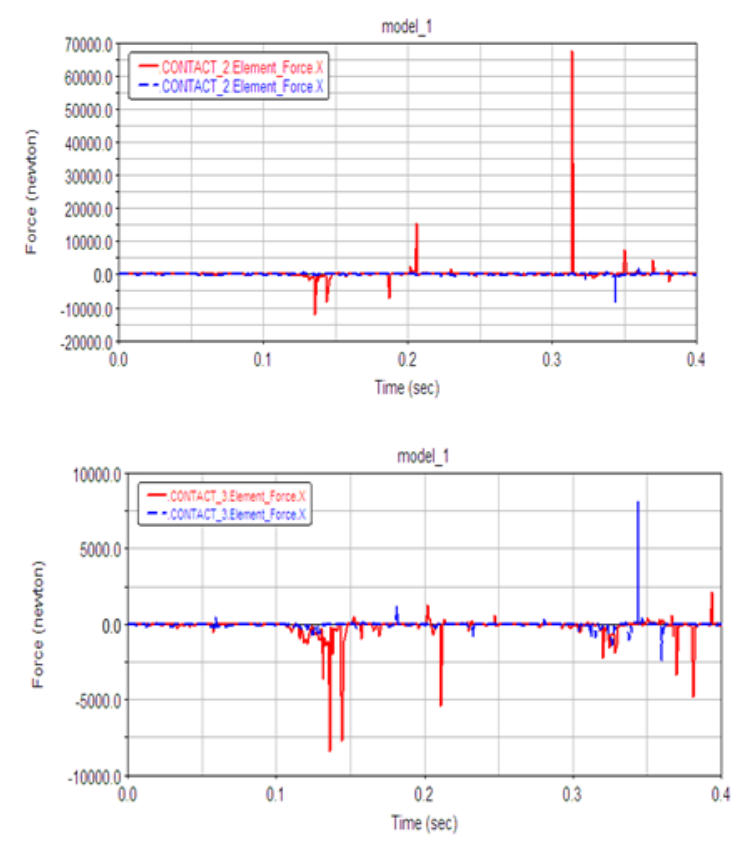

Figure9. X-axis contact force between shaft and sleeve of link and rocker in the case of the rigid and flexible rocker.

Figure10. X-axis contact force between shaft and sleeve of rocker and rack in the case of the rigid and flexible rocker.

\section{Conclusions}

In this paper, including many crank rocker mechanism with clearance joints as the research object, the introduction of flexible factor, rigid flexible rocker rocker and mainly analyzes the influence of the dynamic characteristics of a body. according to the results of the existence of kinematic pair clearance more speed of center of mass of connecting rod and a handle, the acceleration and collision force of the pin shaft and shaft sleeve is especially significant, the influence of clearance on the dynamic stability of the crank rocker mechanism has a very adverse effect. When the rigid rocker into flexible rocker, connecting rod and a handle the velocity and acceleration of wave has improved to some extent, weaken the negative influence of clearance, relatively buffer intensity 
of the collision of pin shaft and shaft sleeve, the mechanism motion more tend to be ideal.

\section{References}

1.Xinglin Guo, Zikun Zhao: Dynamic Characteristics Analysis of the Crank-rocker Mechanisms with Joint Clearance[J], Mechanical Strength, 2010, 32(6):905 909 .

2.Dubinsky S, Freudenstein. Dynamic Analysis of Mechanical Systems with Clearance. Part2:Dynamic Response[J]. ASME, J. Eng. Ind, 1971, 93B(1):305-309.

3.Shaojun Bo,Ruibin Guo: Dynamic Characteristic Research of Planar Four-bar Mechanism with Joint Clearance[C],Advanced in Design Technology,ADME2012,2012.8,Taiyuan, china:912-916.

4.Bo Shaojun, Si Zhenjiu, Ye Qinghuai. Dynamics Simulation Research of Planar Four-bar Mechanism with Multi-joint Clearance[C]/Part1:Applied Mechanics and Materials,ICFMM2014.Guilin:539-542.

5.K.L.Johnson.ContactMechanics[M].Cambridge: Cam-bridge University Press,reprint,1987:92-98. 6.Imed Khemili, Lotfi Romdhane: Dynamic Analysis of Flexible Slider-crank Mechanism with Clearance[J]. European Journal of Mechanics A/Solids, 2008, 27(5):885-886. 